\title{
Rapid Non-Destructive Prediction of Water Activity in Dry-Cured Meat ${ }^{+}$
}

\author{
Alex Mason 1,2, , Magomed Muradov ${ }^{2}$, Badr Abdullah ${ }^{2}$, Ahmed Al-Shamma'a ${ }^{2}$ and \\ Ole Alvseike 1 \\ 1 Animalia, Norwegian Meat Research Institute, 0513 Oslo, Norway; ole.alvseike@animalia.no \\ 2 Faculty of Engineering and Technology, Liverpool John Moores University, Liverpool L3 3AF, UK; \\ m.muradov@ljmu.ac.uk (M.M.); b.m.abdullah@ljmu.ac.uk (B.A.); a.al-shamma@ljmu.ac.uk (A.A.-S.) \\ * Correspondence: alex.mason@animalia.no; Tel.: +47-917-49-792 \\ † Presented at the Eurosensors 2018 Conference, Graz, Austria, 9-12 September 2018. \\ Published: 30 November 2018
}

\begin{abstract}
Water activity (aw) describes the amount of free water available in a matrix for growth of microbiological pathogens and spoilage flora. It is used to predict the safety of food products, and has particular importance for dry-cured meat manufacturers. Results from tests on dry-cured pork $(n=83)$ demonstrate a high degree of correlation $\left(R^{2}=0.909\right)$ with current industry standard equipment. System accuracy at the $95 \%$ confidence interval (0.0125) is comparable with existing equipment available to industry. However, the added advantage of the microwave sensor to enable rapid and non-destructive measurement means that it could be used for day-to-day monitoring and optimization of products within the dry-cured meat value chain. This would reduce per-product operating costs and waste, in addition to facilitating recipe development (e.g., reduced salt).
\end{abstract}

Keywords: microwave spectroscopy; microwave sensor; water activity; non-destructive testing; dry-cured meat

\section{Introduction}

Meat curing is the process by which food products are preserved and flavored, typically through the addition of salts, nitrates, nitrites or sugars [1], and perhaps in combination with other cooking or smoking processes. Despite meat curing being used for hundreds of years to produce stable longlife products, there are few modern techniques available to monitor the curing process in-situ. This leaves producers reliant on rule-of-thumb methods, or measurements of product weight or feel. There has been an increase in the availability and use of sensors in the red meat industry to improve product quality, improve safety and facilitate automation. However, such advancements have had little impact on improving cured meat production, and so heavy reliance on the experience of key workers prevails. For this reason, the authors have been developed a rapid non-invasive tool for the measurement of water activity $\left(\mathrm{a}_{\mathrm{w}}\right)$, which operates using a microwave-based sensing system.

Water activity is a classic parameter, ranging between 0 and 1 , describing the amount of free water available in a matrix for growth of microbiological pathogens and spoilage flora. It is used to predict the safety of food products, and for dry-cured meat manufacturers it is the most important control parameter. There are devices available on the market today to measure aw, with the market leaders being Meter Group (formerly Aqualab), Novasina and Rotronic. However, the devices are typically characterized by slow (5-30 $\mathrm{min}$ ) measurement speeds and their destructive or invasive implementations. Moreover, most of the devices are better suited to a laboratory environment than at the production line. In combination, these characteristics typically limit measurements to the minimum required to ensure safety standards are met due to the economic loss incurred in 
destructive testing. Furthermore, they do little to help optimize the curing process itself. Introduction of rapid non-invasive measurement tools would be beneficial for a number of reasons, including; process optimization; higher safety standards; lower per-unit production costs; recipe development (including salt reduction); and reduced waste.

\section{The Microwave Sensor}

The device tested during this work is based on a microwave sensing technique, whereby microwave energy is radiated from a probe, and the change in signal at selected frequencies when exposed to a cured meat product is analyzed. The authors have demonstrated the technique previously for invasive measurement of $\mathrm{a}_{\mathrm{w}}[2]$ and water holding capacity [3] in the meat industry, as well as in other sectors such as medical and water; for non-invasive measurement of lactate [4], as well as in-situ detection of antibiotics [5] in aquaculture and heavy metals [6] in polluted mine waters. The concept for measuring aw has been patented (United Kingdom Patent Application No. 1515498.2).

The system consists of a probe, some means to generate microwave energy, and a system for analyzing the return microwave signal to predict aw. During validation of this technique a Vector Network Analyser (Rohde \& Schwarz, Oslo, Norway), or VNA for short, is used to generate microwave spectra and detect corresponding changes. The sensor is a single port device, connecting to the VNA via a coaxial cable. The probe is designed such that the user must hold the sensitive end against the measurement target with minimal pressure. After a few seconds, sufficient data is acquired to predict the aw. The probe is illustrated in Figure 1, both alone and in example use at a production plant in Spain. While the original idea for this device was monitoring high-value curedhams, it is anticipated that the same principle can be applied to other meat products, including sausages. Furthermore, on-going work shows that the device can be used successfully on products in vacuum wrapping which broadens its potential for use throughout the dry-cured meat value chain.

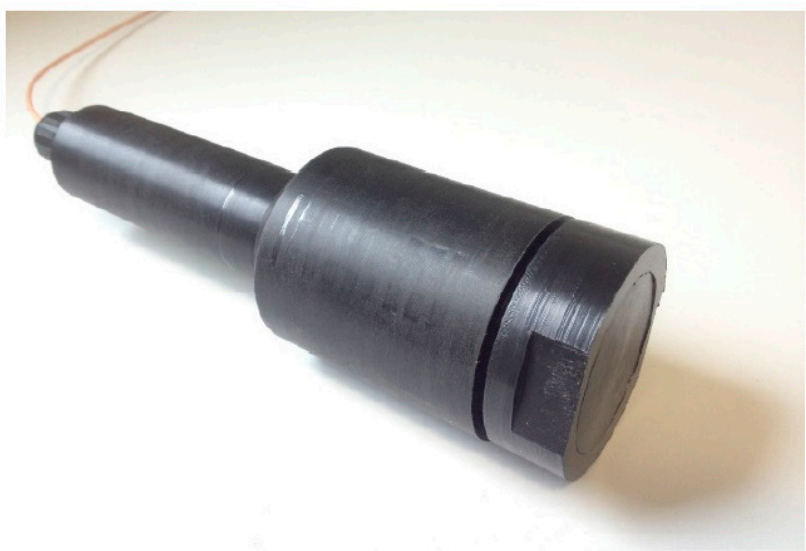

(a)

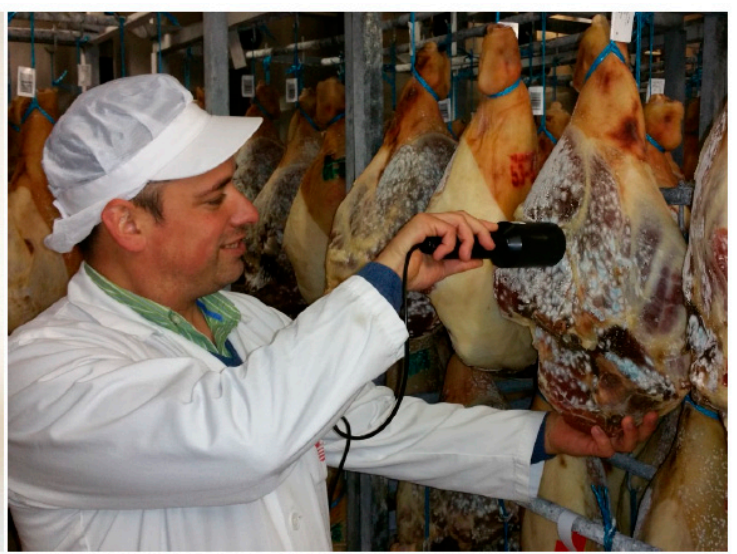

(b)

Figure 1. (a) The microwave probe and (b) the probe demonstrated at dry-cured production facility in Spain.

\section{Methodology}

Pork meat samples $(n=83)$ were measured during curing. Pork loins were sourced from a local slaughter house $24 \mathrm{~h}$ post-slaughter. They were cut into uniform pieces, salted, sealed in vacuum bags and stored for 2 weeks to allow equalization. The samples were then dry-cured in a temperature stable environment $\left(12-14{ }^{\circ} \mathrm{C}, 72-74 \%\right.$ humidity) until weight loss was $40 \%$ of the initial weight. Typically, industry aims for 30-35\% weight loss, but this was exceeded to further increase the range of aw values observed. For further detail on the preparation method, see Bjarnadottir et al. [2]. Samples were measured throughout the curing period, firstly with the microwave system (see Section 2) and then with an industry standard aw meter (Aqua Lab AS, Bergen, Norway). Due to 
the large number of samples required to enable comparison with the standard (destructive) aw meter, preparation and measurement took place in batches over a period of approximately 6 months.

Captured spectral data was normalized as selected frequencies, with this then being analyzed using Partial Least Squares (PLS) regression. The PLS technique was selected due to its ability to handle large numbers of independent variables with multi-collinearity-each spectrum consisted of 4000 discrete frequencies. PLS regression analysis was carried out using MATLAB libraries (libPLS) written by Li, Xu and Liang [7].

\section{Results}

Observed aw measurements, using a desktop meter, varied between 0.969 (fresh samples) and 0.749 (max. dry-cured). From a safety perspective, $a_{w} \leq 0.86$ is considered acceptable for vacuum sealed products with a modified atmosphere [1].

At selected frequencies, a high degree of correlation was observed between the aw and sensor output. Figure 2a illustrates this correlation, where adjusted $R^{2}=0.909$. PLS regression shows the ability of the sensor to predict aw based on measured values, as in Figure $2 \mathrm{~b}$. Accuracy (mean = $0.0141,95 \%$ confidence $=0.0125$, max. $=0.0488$ ) is lower than the benchmark equipment used in this work, which is stated to be 0.01 by the manufacturer. It is however comparable with less expensive devices from Meter Group (Aqualab Pawkit, accuracy = 0.02), Novasina (Labmaster-aw, accuracy = 0.03 ) and Rotronics (Hygropalm HP23-aw, accuracy $=0.05$ ). The reason for the reduced accuracy is most likely due to the deformation of samples during the curing process; as illustrated in Figure 3, surfaces become hard and uneven, and so finding a suitably flat surface for good contact with a sample becomes more difficult. This may be overcome in the future through altering the probe design to better accommodate uneven and curved surfaces.

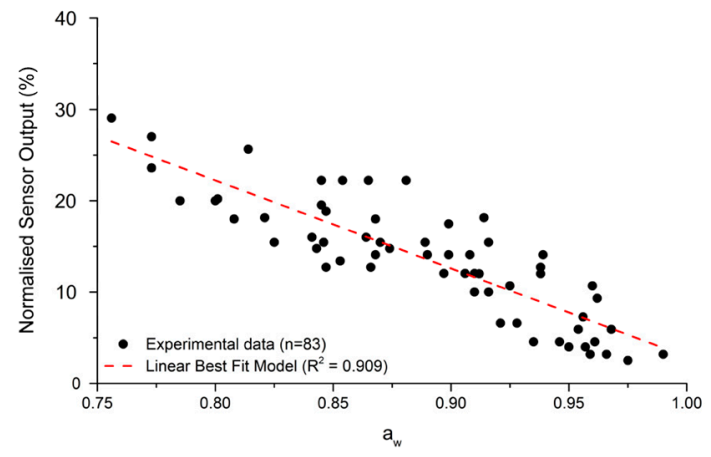

(a)

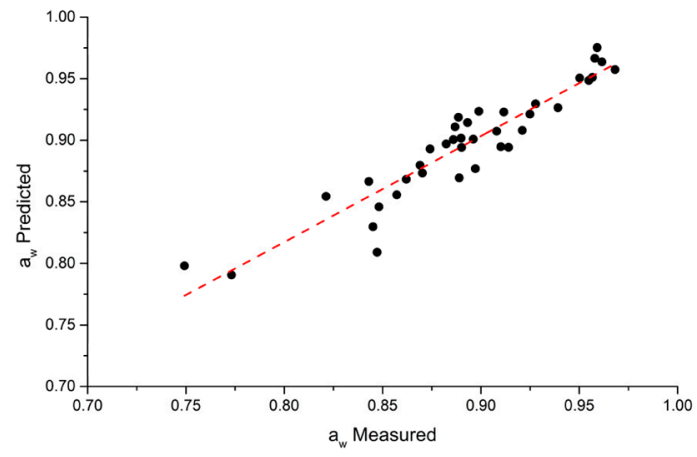

(b)

Figure 2. (a) Correlation between the aw and normalized sensor output at selected frequencies. PLS regression (b) shows the capability of the sensor in terms of measured vs. predicted aw values.

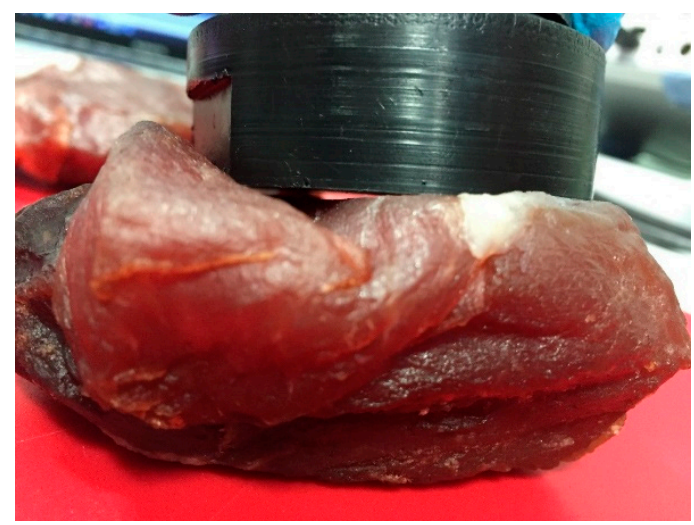

Figure 3. Uneven surfaces, which are more prevalent as the product dries and hardens, introduce gaps between the sensitive surface of the microwave probe and the sample. 


\section{Conclusions}

This paper reports on the testing of a novel microwave-based sensor for measuring water activity. Current methods for measurement are typically slow and destructive, which limits the use of aw for optimizing dry-cured meat production. Results from tests on dry-cured pork $(n=83)$ demonstrated a high degree of correlation $\left(R^{2}=0.909\right)$ with current industry standard equipment. System accuracy at the $95 \%$ confidence interval (0.0125) is comparable with existing equipment available to industry. However, the added advantage of the microwave sensor to enable rapid and non-destructive measurement means that it could be used for day-to-day monitoring and optimization of products within the dry-cured meat value chain. This would reduce per-product operating costs and waste, in addition to facilitating recipe development (e.g., reduced salt). The potential in shelf-stable non-meat products will also be considered in the future.

Author Contributions: A.M., O.A. and A.A.-S. conceived the original idea and implemented an appropriate design for the sensor and concept. B.A. assisted with preliminary testing and fabrication of the probe housing. M.M. implemented the experimental procedure and reported the findings. Writing of this paper was undertaken by A.M.

Acknowledgments: The authors would like to thank Prolongo (Malaga, Spain), in particular Francisco Requena, for their support and contributions during this project.

Conflicts of Interest: The authors declare no conflict of interest.

\section{References}

1. Taormina, P.J. Meat and poultry / curing of meat. In Encyclopedia of Food Microbiology, 2nd ed.; Batt, C.A., Tortorello, M.L., Eds.; Academic Press: Oxford, UK, 2014; pp. 501-507.

2. Bjarnadottir, S.G.; Lunde, K.; Alvseike, O.; Mason, A.; Al-Shamma'a, A.I. Assessing quality parameters in dry-cured ham using microwave spectroscopy. Meat Sci. 2015, 108, 109-114.

3. Mason, A.; Abdullah, B.; Muradov, M.; Korostynska, O.; Al-Shamma'a, A.; Bjarnadottir, S.G.; Lunde, K.; Alvseike, $\mathrm{O}$. Theoretical basis and application for measuring pork loin drip loss using microwave spectroscopy. Sensors 2016, 16, 182.

4. Mason, A.; Korostynska, O.; Louis, J.; Cordova-Lopez, L.E.; Abdullah, B.; Greene, J.; Connell, R.; Hopkins, J. Noninvasive in-situ measurement of blood lactate using microwave sensors. IEEE Trans. Biomed. Eng. 2018, 65, 698-705.

5. Korostynska, O.; Jansomboon, W.; Nakouti, I.; Mason, A.; Al-Shamma'a, A. Tackling water pollution: Realtime monitoring of residual antimicrobials concentration in aquaculture with microwave spectroscopy. Adv. Mater. Sci. 2018, 3, 1-6.

6. Frau, I.; Korostynska, O.; Mason, A.; Byrne, P. Comparison of electromagnetic wave sensors with optical and low-frequency spectroscopy methods for real-time monitoring of lead concentrations in mine water. Mine Water Environ. 2018, 37, 617-624.

7. Li, H.-D.; Xu, Q.-S.; Liang, Y.-Z. Libpls: An integrated library for partial least squares regression and linear discriminant analysis. Chemom. Intell. Lab. Syst. 2018, 176, 34-43.

(C) 2018 by the authors. Submitted for possible open access publication under the terms and conditions of the Creative Commons Attribution (CC BY) license (http://creativecommons.org/licenses/by/4.0/). 